\title{
exploitation rationnelle d'un terril
}

\author{
par \\ C. Schroeder \\ Université de Liège (Belgique)
}

RESUME - L'exploitation des crassiers ou "terrils" anciens, à teneur suffisante en charbon permet la production économique de matière énergétique associée à une action de rénovation des sites. Dans la région de Charleroi (Belgique), une société spécialisée, la S.A. Ryan Europe, compte prochainement exploiter le terril "6 Perier" et se servir des résidus comme matériaux de remblais pour l'assainissement d'une vallée marécageuse adjacente.

Le terril ayant antérieurement glissé et causé d'importants dégâts, une étude géologique, hydrogéologique et géotechnique a conduit à définir les caractéristiques du site et à préconiser les modes d'exploitation et les conditions d'exécution des remblais offrant les garanties de sécurité.

\section{INTRODUCTION}

Une des caractéristiques majeures, sinon la principale, du paysage de la région de Charleroi en Belgique est la profusion de terrils ou crassiers, constitués des stériles de charbonnages accumulés depuis le siècle dernier. Les techniques anciennes de lavage de la houille ne permettaient pas d' extraire la totalité du charbon et les terrils de l'époque contiennent encore une notable proportion de combustible (jusqu'à 10\%, voire plus).

L'augmentation du coût de l'énergie rend leur exploitation rentable. Pour cela, les dépôts de terrils sont traités par divers procédés séparant le charbon des stériles, schisteux principalement. En général, après le traitemont, les résidus sont redéposés au même site et constituent un terril "remodelé". Les opérations de remodelage peuvent être conduites de manière à améliorer l'aspect du site, à procéder au reboisement, à en augmenter la stabilité et à le valoriser pour d'autres usages : espaces verts, lotissements, etc...

\section{LE TERRIL "6 PERIER"}

La S.A. Ryan Europe, spécialisée dans ces travaux et ayant acquis une grande expérience outre-manche, exploite de la sorte un certain nombre de terrils dans la région de Charleroi. Elle se propose actuellement de mettre en exploitation le terril "6 Perier" présentant des caractéristiques remarquables tant par les problèmes de stabilité qu'il pose que par les possibilités d'utilisation optimum des résidus aux fins d'amélioration du site.

Le terril (fig. 1 p. 2) est situé à Courcelles, commune voisine de Charleroi et appartient aux charbonnages de Monceau-Fontaine. L'édification du terril débute en 1895. En 1926, un glissement refoule d'une quinzaine de mètres, sur $120 \mathrm{~m}$ de longueur, le chemin au Nord-Est du terril. Les dépôts sont alors arrêtés de ce côté.

En 1938, un important glissement détruit plusieurs immeubles au Nord du terril. Actuellement, des bourrelets périphériques marginaux témoignent encore des mouvements de 1938. 


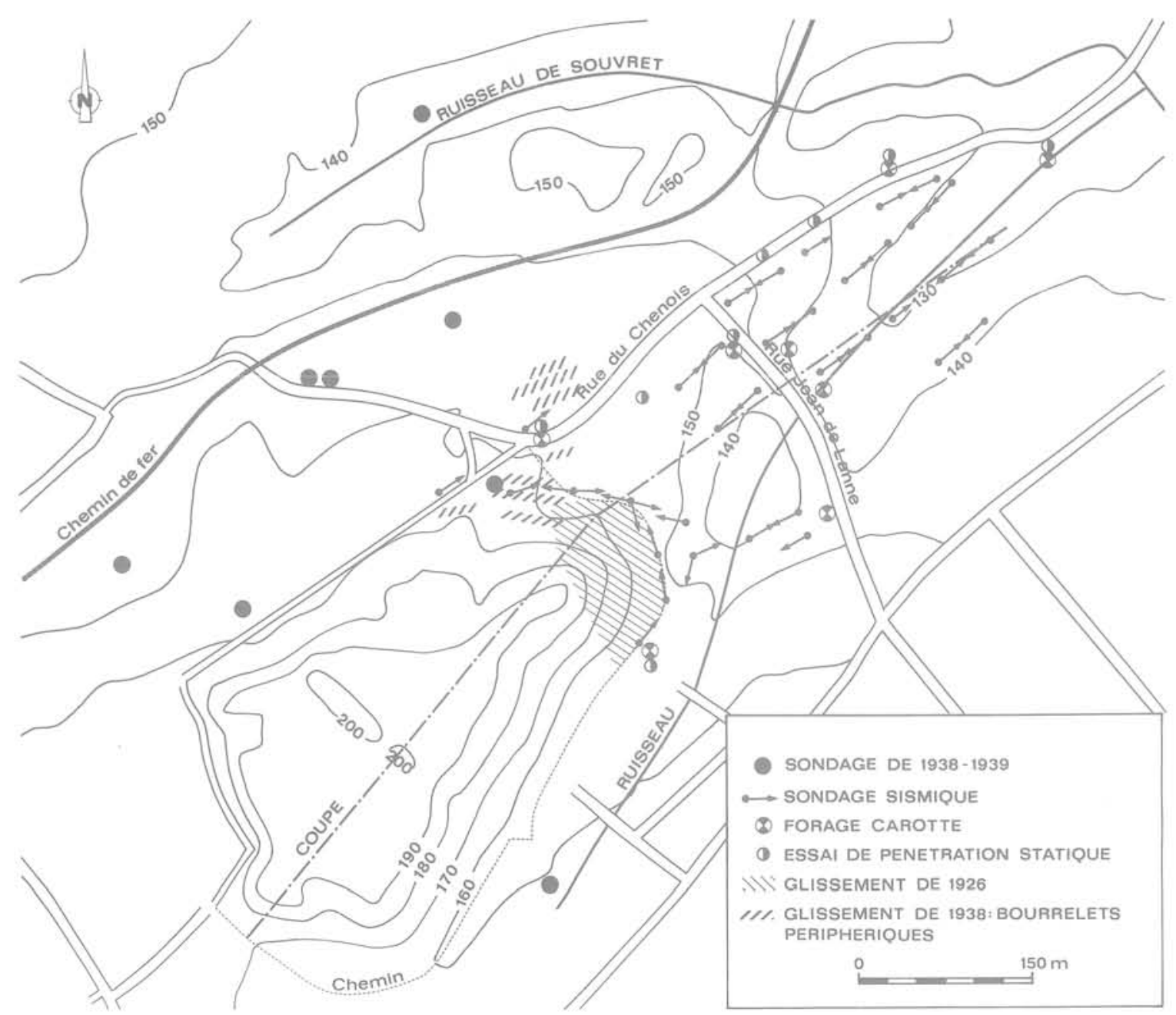

Fig. 1 - Situation du terril "6 Perier"
- Reconnaissance géologique

Après le glissement de 1938, le site a été abandonné, le volume total du terril étant alors de l'ordre de $1.800 .000 \mathrm{~m}^{3}$.

Au Nord-Est se trouve la vallée marécageuse d'un affluent du ruisseau du Moulin de Souvret. Dans le cadre d'un projet d'assainissement du site, il est envisagé de combler la vallée au moyen d'une partie du terril, soit environ $400.000 \mathrm{~m}^{3}$.

La situation présente donc un intérêt économique exceptionnel : l'exploitation étant de toutes manières rentable, il suffit d'en profiter pour redéposer les stériles dans la vallée voisine plutôt qu'au site même du terril. On réalise ainsi l'assainissement à un coût intéressant et en outre on libère une superficie de $1^{\prime}$ ordre de 1 à 2 hectares occupée par l'ancien terril.

Le solde non exploité et la partie remodelée seraient conservés comme zone boisée et témoin du passé industriel de la région.

\section{RECONNAISSANCES GEOLOGIQUE, HYDROGEOLOGIQUE ET GEOTECHNIQUE}

Nos recherches ont comporté, outre un inventaire des connaissances (sondages, archives et publications), une campagne géophysique par sismique-réfraction ( 40 sondages), huit forages mécaniques équipés en piézomètres, avec prélèvement d'échantillons non remaniés et essais de laboratoire et huit essais de pénétration statique.

Le promontoire sur lequel est établi le terril peut être schématiquement décrit comme suit, de haut en bas (fig. 2 p. 4) :

- des terrains quaternaires, sables limoneux et limons, épais de 2 à $5 \mathrm{~m}$ sur les flancs de la vallée et pouvant atteindre 6 à $7 \mathrm{~m}$ dans le fond.

- des sables argileux bruxelliens, parfois peu denses mais, dans l'ensemble, de bonne tenue. Leur base est subhorizontale à la cote 158 à 160 . 
- des argiles et argiles sableuses yprésiennes, épaisses localement de plus de $10 \mathrm{~m}$

- le bed-rock houiller, schisto-gréseux, décomprimé et altéré sur une épaisseur de plus de $5 \mathrm{~m}$.

A l'Est de la rue Jean de Lanne, l'érosion a éliminé les formations tertiaires et on rencontre directement le Houiller sous les formations superficielles.

Les caractéristiques géomécaniques des formations ont été mesurées in situ et en laboratoires.

- In situ, la réalisation simultanée en quelques points d'essais de pénétration, d'essais sismiques et de forages carottés a permis de définir pour chaque type de terrain les valeurs de la résistance à la pointe $\mathrm{Rp}$, du frottement latéral $F_{1}$ et de la vitesse des ondes sismiques longitudinales $V_{L}$ :

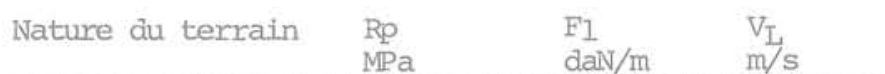

1.remblais irrégulière irrégulier 300

2. quaternaire

limon de surface

argile limoneuse

$\begin{array}{crl}1,5 & 0 & 350 \\ \text { variable } & 370 & 1000 \\ (1,5-3,0) & & \end{array}$

3. Bruxellien

sable avec concré-

tions

4.Yprésien

argile

argile compacte

irrégulière irrégulier 1000

$(2,5)$

. Houiller

schistes altérés

1,5

variable

250

700

1000

$(2,0-6,0)$

schistes

gréseux altérés

variable

625

$(2,0-20,0)$

bed rock sain

$\begin{array}{rl}20 & 3000 \\ \text { refus } & \text { id. }\end{array}$

id.

1500 2000 à 4000

On remarque ainsi que les limons de surface quaternaires ont des caractéristiques assez moyennes ( $R p \bumpeq 1,5 \mathrm{MPa} \max$.$) . Dans le bed$ rock altéré, la vitesse des ondes sismiques est très faible (moins đe $1000 \mathrm{~m} / \mathrm{s}$ ), ce qui empêche son repérage sous l'argile yprésienne compacte $\left(V_{L}=1800 \mathrm{~m} / \mathrm{s}\right)$; les essais de pénétration en montrent cependant la bonne tenue mécanique.

- Au laboratoire, les échantillons non remaniés ont fait l'objet de tests d'identification, d'essais oedométriques (pour déterminer une éventuelle gonflance), d'essais triaxiaux $\mathrm{Cu}_{\mathrm{u}}$ avec mesure de la pression interstitielle et d'essais de compression simple pour la détermination de $\mathrm{C}_{\mathrm{u}}$ de $\mathrm{I}^{\prime}$ argile compacte. Seules les formations quaternaires et yprésiennes ont été testées, le Bruxellien n'intervenant pas dans la zone à remblayer.

Les limons quaternaires ont une cohésion à peu près nulle mais l'angle de frottement interne avoisine les $35^{\circ}$; la compressibilité et le gonflement sont assez faibles (module de compressibilité $\mathrm{C}=45, \mathrm{~A}=441$ ). Ils sont peu perméables : $\mathrm{k}=4 \cdot 10^{-9} \mathrm{~m} / \mathrm{s}$.

Les formations yprésiennes sont caractérisées en moyenne par les valeurs effectives suivantes : $\quad \mathrm{c}^{\prime}=24$ à $62 \mathrm{kPa}$

$\phi^{\prime}=14,5^{\circ}$ à $16^{\circ}$; les coefficients $C$ et $A$ valant respectivement de 14 à 36 et de 57 à 220, ce qui indique une faible gonflance.

L'argile yprésienne de base est moyennement cohérente $(\mathrm{Cu}=64,4 \mathrm{kPa})$, assez compressible $(C=24)$ et un peu gonflante $(A=65)$. Elle est imperméable.

En ce qui concerne les dépôts du terril proprement dit, ils ont un angle de talus naturel de $30^{\circ}$ environ, une cohésion faible et leur masse volumique sèche en surface est de I'ordre de $1530 \mathrm{~kg} / \mathrm{m}^{3}$.

L'étude hydrogéologique a montré que le terril présente une perméabilité élevée. Les eaux de pluie s'y infiltrent, cheminent dans la vallée enfouie et ressortent par une source dont le niveau est légèrement au-dessus de l'ancien fond de la vallée, celui-ci ayant été soulevé par les mouvements de terrain de 1926 .

Dans le substratum, on distingue la nappe des sables bruxelliens et yprésiens et celle du bed rock houiller. La première est alimentée par la nappe du terril et son exutoire se situe à la recoupe du toit des argiles yprésiennes (imperméables) par la surface topographique. La seconde est une nappe de fissures s'écoulant vers le ruisseau qui constitue son niveau de base; elle engendre les zones marécageuses du fond de la vallée. Comme il est de règle dans les nappes de fissures du terrain houiller, les gradients piézométriques sont élevés et les fluctuations importantes suivant les taux d'infiltration.

\section{STABILITE ET SECURITE}

Les résultats des essais de laboratoire permettent d'expliquer les raisons du glissement de 1938. Il s'agit d'une rupture par défaut de force portante ou plutôt d'une rupture de remblais sur sol "mou", en 1'occurrence $1^{\prime}$ argile yprésienne, la surface de rupture étant vraisemblablement tangente au substratum houiller, ce qui nous place dans les conditions d'utilisation des abaques de calcul.

En fonction des résultats des essais, on a calculé la stabilité du terril lors du déchargement. Pour éviter que les excavations ne réactivent le glissement et pour obtenir à court et à long terme une sécurité suffisante (coefficient de sécurité $F \bumpeq 1,5)$, on a été amené à préconiser un mode d'exploitation en gradins donnant au terril une pente globale de $20^{\circ}$ environ, alors que la pente actuelle est de $30^{\circ}$. 


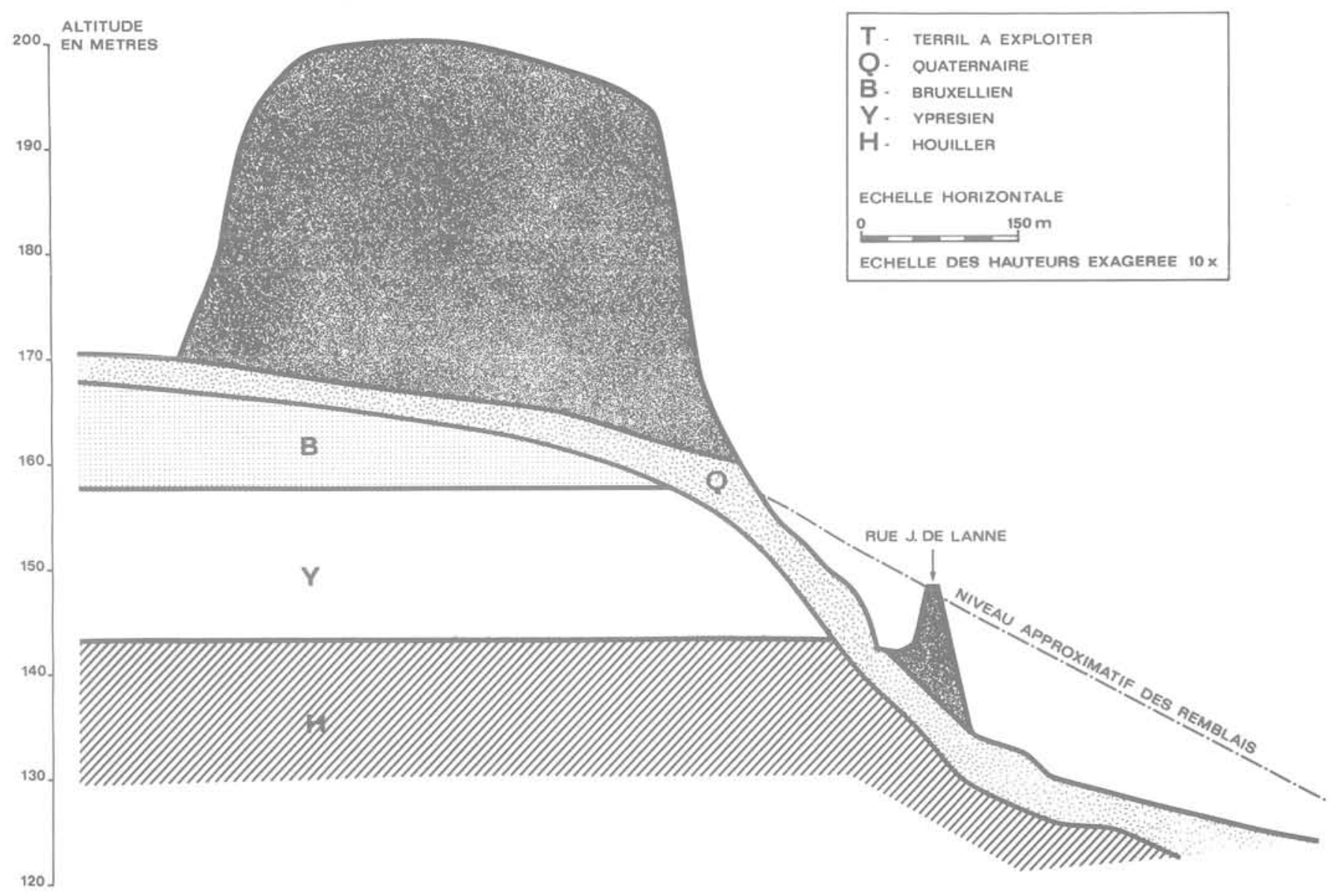

Fig. 2 - Coupe géologique schématique Sud-Ouest/Nord-Est

La stabilité propre des matériaux mis en remblais est assurée par la géométrie des đépôts : remblais de faible épaisseur $(12 \mathrm{~m}$ maximum) déposés dans une cuvette à faible pente $\left(7^{\circ}\right.$ à $\left.10^{\circ}\right)$, comme $1^{\prime}$ indique la fig. 2 .

Compte tenu de la valeur de l'angle de frottement interne des formations limoneuses $\left(35^{\circ}\right.$ maximum), la stabilité d'ensemble des remblais sur la couche limoneuse tapissant la vallée est assurée sauf si le niveau piézométrique s'élève de plus de $10 \mathrm{~m}$ environ au-dessus du niveau topographique initial; 1 'augmentation de pression interstitielle qui en résulterait amènerait le coefficient de sécurité du glissement sur la couche de limons à des valeurs inférieures à l'unité.

La mise en pression pourrait provenir d'un colmatage du collecteur traversant la rue Jean de Lanne qui "barre" la vallée, ce qui. provoquerait dans le remblais la formation d'une nappe pouvant atteindre $12 \mathrm{~m}$ de niveau. Il est aisé d'éviter ce colmatage (construction de chambres de visites et entretien régulier). D'autre part, la nappe du bed rock houiller trouvait un exutoire dans le fond de la vallée, par ailleurs marécageux; les limons comprimés sous le poids des remblais verront leur perméabilité diminuer et la nappe des fissures du Houiller ne trouvant plus son exutoire normal risque de se mettre en charge. Ce phénomène est susceptible d'exéder la dizaine de mètres, compte tenu de la topographie des lieux. La solution préconisée est de réaliser dans les flancs de la vallée à combler, des drains longitudinaux traversant la couche quaternaire et atteignant le bed rock de manière à recréer un exutoire à la nappe du Houiller.

\section{CONCLUSIONS}

L'étude géologique, hydrogéologique et géotechnique du site a permis de définir avec précision la nature et la géométrie des terrains, leurs caractéristiques mécaniques et le régime des eaux souterraines.

On en a déduit les conditions de l'exploitation et des mises en dépôt des stériles pour garantir la sécurité tant en phase d'exploitation qu'ultérieurement.

L'exploitation du terril "6 Perier" permettra un assainissement économique de la zone marécageuse voisine puisque les remblais, ainsi que leur mise en place, ne sont que les sous-produits, les résidus obligatoirement remis en dépôt, de l'opération de récupération des matières premières énergétiques subsistantes. 
BREYER F. (1962), Note concernant le terril du siège Saint-Nicolas de la S.A. des Charbonnages Espérance et Bonne Fortune, Technique et Humanisme (1) 31-41, Liège.

CALEMBERT L., DANTINNE R. (1965), L'avalanche de cendres volantes survenues à Jupille (Liège) le 3 février 1961, Volume d'hommage au Prof. F. CAMPUS, 41-57, Liège.

FONTAINE A. (1938), Constatations parallèles, au début de 1938 , sur les versants artificiels et naturels de Schepdael, la Fléchère, Courcelles et Eigenbilsen, Bull. Société Belge de Géologie, de Paléontologie et d'Hydrogéologie (48), 155-169, Liège.

LINARD DE GUERTECHIN A. (1939), Action tectonique des terrils. Glissements de Courcelles et de Fontaine-L'Evêque, Bulletin technique de l'Union des Ingénieurs sortis des Ecoles Spéciales de Louvain, 39-61, Louvain.
MARLIERE R. (1950), Terrils "en marche", Bulletin A.I.M.S. $1950(4), 11-18$, Mons.

MONJOIE A., SCHROEDER C. (1978), Auscultation des massifs rocheux par sismiqueréfraction et propriétés géođynamiques des terrains, Proc. III, Int. Congrès I.A.E.G. Sec. IV (2) 29-36, Madrid.

PILOT G. MOREAU M. (1973), La stabilité des remblais sur sols mous. Abaques de calcul. Ed. Eyrolles, Paris.

Rapports inédits des Laboratoires de Géologie de 1 'Ingénieur et d'Hydrogéologie. 